\title{
VERTICES OF LOCALIZED IMBALANCE IN A BIASED GRAPH
}

\author{
THOMAS ZASLAVSKY
}

\begin{abstract}
A biased graph consists of a graph $\Gamma$ and a subclass $B$ of the polygons of $\Gamma$, such that no theta subgraph of $\Gamma$ contains exactly two members of $B$. A subgraph is balanced when all its polygons belong to $B$. A vertex is a balancing vertex if deleting it leaves a balanced graph. We give a construction for unbalanced biased graphs having a balancing vertex and we show that an unbalanced biased graph having more than one balancing vertex is an unbalanced series or parallel connection of balanced graphs.
\end{abstract}

Introduction. A polygon in a graph $\Gamma$ is the edge set of a simple closed path. A subclass of polygons of $\Gamma$ is a linear subclass if no theta subgraph of $\Gamma$ contains exactly two polygons in the subclass. A biased graph consists of a graph $\Gamma$ and a linear subclass $B$ of polygons of $\Gamma[2]$. A subgraph or edge set is balanced if every polygon in it belongs to $B$. Biased graphs are a generalization of ordinary graphs, in the sense that for most purposes the latter can be treated as biased graphs that are balanced. For instance, a biased graph $(\Gamma, B)$ has a "bias matroid" $G(\Gamma, B)$ and a "lift matroid" $L(\Gamma, B)$ (see $[3]$ ), which when $(\Gamma, B)$ is balanced equal the usual polygon matroid of $\Gamma$.

We say that the imbalance of a biased graph $(\Gamma, B)$ is localized at a vertex $v$ if the vertex-deleted subgraph $\Gamma \backslash v$ is balanced. Briefly, we call $v$ a balancing vertex. We are interested in biased graphs that have balancing vertices because in several senses they are among the least unbalanced. For one thing, by definition they are the unbalanced graphs in which balance results by deleting the fewest vertices. They are also the simplest kind of unbalanced graph having no two vertex-disjoint unbalanced polygons. Finally, they are among those biased graphs whose bias and lift matroids are graphic matroids.

In this article we see that the set $V^{*}$ of balancing vertices of $(\Gamma, B)$ is usually empty and we describe the exceptions (see the Theorem and Corollary 2).

Definitions and a lemma. We let $\Gamma$ denote a graph $(V, E)$ with vertex set $V$ and edge set $E$. All graphs are finite. Loops and multiple edges are allowed. By $m K_{2}$ we mean $m$ links (edges with two distinct endpoints) in parallel on two vertices. By the polygon graph $C_{l}$ we mean the graph of a simple, closed path of length $l$; in particular, $C_{2}=2 K_{2}$.

Received by the editors November 20, 1985 and, in revised form, June 14, 1986.

1980 Mathematics Subject Classification (1985 Revision). Primary 05C99; Secondary 05C25.

Key words and phrases. Biased graph, balancing vertex, signed graph.

Research assisted by National Science Foundation grant DMS-8407102. The author was a Visiting Research Fellow, 1984-1985, at the University of Evansville. 
We let $\Phi=(\Gamma, B)$ denote a biased graph whose underlying graph is $\Gamma$. We call it contrabalanced if $B=\varnothing$. The biased union $\Phi_{1} \sqcup \Phi_{2}$ of biased graphs is their edge-disjoint graph union $\left(V_{1} \cup V_{2}, E_{1} \cup E_{2}\right)$ with the balanced polygon class $B_{1} \cup B_{2}$. The contraction $\Phi / \Delta$ of $\Phi$ by a balanced and connected subgraph $\Delta$ is the usual contraction graph $\Gamma / \Delta$ (where $V(\Delta)$ becomes a vertex and the edges of $\Delta$ are discarded) with the balanced polygon class

$\{C \in B: C \subseteq E \backslash E(\Delta)$ and $C$ is a polygon in $\Gamma / \Delta\}$

$$
\cup\{C \backslash E(\Delta): C \in B \text { and } C \cap E(\Delta) \text { is a simple open path }\} \text {. }
$$

(This definition is drawn from [2], where arbitrary contractions are defined.)

In $\Gamma$ the bridges of a vertex set $U \subseteq V$ are the maximal connected subgraphs $\Delta$ meeting $U$ and such that $\Delta$ is connected through $V \backslash U$; we count an edge with endpoints in $U$ as a bridge [1]. We call $\Delta$ a $k$-bridge if it contains at least $k$ vertices of $U$. A bridge of a vertex $v$ is merely a bridge of $\{v\}$. A cutpoint is a vertex with more than one bridge. A block of $\Gamma$ is a maximal subgraph with no cutpoint. For instance, a loop is a block. A cutpair is a pair of vertices lying in a common block and having in that block at least two bridges. (This is nonstandard. Usually one requires some sort of nontriviality.) We apply the same terminology to a biased graph, ignoring its bias.

Let $\Gamma$ and $\Gamma_{1}$ be two graphs and let $e \in E, e_{1} \in E_{1}$ be links; we require that $|E|,\left|E_{1}\right| \geq 2$. The 2-sum of $\Gamma$ and $\Gamma_{1}$ at $e$ and $e_{1}$ is obtained in the following way. We choose isomorphic copies of $\Gamma$ and $\Gamma_{1}$, if necessary, to assure that the vertices of $e$ and $e_{1}$ are the same but $\Gamma$ and $\Gamma_{1}$ have nothing else in common. The 2-sum is the union $\Gamma \cup \Gamma_{1}$ with $e$ and $e_{1}$ deleted. We may say $\Gamma_{1}$ is 2 -summed into $\Gamma$ at $e_{1} \in E_{1}$ (and at $e \in E$ ).

Suppose $\Phi=(\Gamma, B)$ and $\Phi_{1}=\left(\Gamma_{1}, B_{1}\right)$ are biased graphs. Their 2-sum at $e_{1} \in E_{1}$ and $e \in E$ is the 2-sum of $\Gamma$ and $\Gamma_{1}$ with the balanced polygon class

$B \cup B_{1} \cup\left\{P \cup P_{1} \subseteq\left(E \cup E_{1}\right) \backslash\left\{e, e_{1}\right\}: P \cup P_{1}\right.$ is a polygon and

$$
\left.P \cup e \text { and } P_{1} \cup e_{1} \text { are balanced polygons }\right\} \text {. }
$$

Let us say two polygons have equal (or similar) balance (or other synonyms) if both are balanced or both are unbalanced.

LEMMA 1. Let $\Phi$ be a biased graph and let $u, v \in V(\Phi)$ be a cutpair whose bridges are balanced. Let $P_{i}, Q_{i}$ be simple paths from $u$ to $v$ in the ith bridge. Then for each $i \neq j, P_{i} \cup P_{j}$ and $Q_{i} \cup Q_{j}$ have equal balance.

ProOF. We may take $P_{j}=Q_{j}$, for this case implies the lemma. For fixed $P_{i}$ we argue by descent on the length $l\left(Q_{i}\right)$ of the initial segment common to $P_{i}$ and $Q_{i}$ in travelling from $u$ to $v$. We may assume $P_{i} \neq Q_{i}$.

Let $x$ be the first vertex after which $P_{i}$ and $Q_{i}$ diverge and let $y$ be the first vertex at which $P_{i}$ again meets $Q_{i}$. Let $R_{i}$ consist of the path $P_{i}$ from $u$ to $y$ and $Q_{i}$ from then on to $v$. Clearly, $l\left(R_{i}\right)>l\left(Q_{i}\right)$, so inductively $P_{i} \cup P_{j}$ and $R_{i} \cup P_{j}$ have similar balance. Since $Q_{i} \cup R_{i} \cup P_{j}$ is a theta graph in which the polygon formed in $Q_{i} \cup R_{i}$ is balanced, the two remaining polygons, that is $Q_{i} \cup P_{j}$ and $R_{i} \cup P_{j}$, are equally balanced. The lemma follows.

We remark that for gain graphs [2] (called "voltage graphs" in [5]) Lemma 1 has a one-line proof based on potential functions. But to treat biased graphs in general it seems to be necessary to use the stepwise "homotopy" method of our proof. 
The structure at a balancing vertex. We can give a construction for biased graphs whose imbalance is localized at a vertex.

PROPOSITION 1. Let $\Phi$ be a biased graph and $v$ a vertex. The vertex-deleted subgraph $\Phi \backslash v$ is balanced if and only if $\Phi$ has the form $(\Psi \sqcup T) / T$, where $\Psi$ and $T$ are balanced biased graphs, $T$ is connected, and $V(T) \subseteq V(\Psi)$. (Then $v$ in $\Phi$ corresponds to the vertex to which $T$ is contracted in $(\Psi \sqcup T) / T$.)

We note that it alway suffices to let $T$ be a tree.

ProOF. First, we note that, if $\Phi$ has the stated form, then $\Phi \backslash v=\Psi \backslash V(T)$, which is balanced.

For the converse, let $\Phi$ be biased and $v$ be a vertex such that $\Omega=\Phi \backslash v$ is balanced. Let $\Omega_{1}, \ldots, \Omega_{r}$ be the connected components of $\Omega$ and let $E_{i}$ be the set of links from $v$ to $\Omega_{i}$. If $e \in E_{i}$, let $w_{e}$ be its vertex other than $v$. We define an equivalence relation $\sim$ on each set $E_{i}$ by $e \sim f$ if $\Omega_{i} \cup(V,\{e, f\})$ is a balanced subgraph of $\Phi$. To prove this is an equivalence relation, suppose $e \sim f \sim g$ in $E_{i}$. Let $P$ be a simple path from $e$ to $g$ in $\Omega_{i}$ and let $Q$ be a simple path from $f$ to $P$ in $\Omega_{i}$ such that $P \cup Q \cup\{e, f, g\}$ is a theta graph. Let $P_{e}$ be the part of $P$ from $e$ to $Q$ and $P_{g}$ the part from $Q$ to $g$. By hypothesis $P_{e} \cup Q \cup\{e, f\}$ and $P_{g} \cup Q \cup\{f, g\}$ are balanced; consequently, $P \cup\{e, g\}$ is balanced. We conclude that $e \sim g$. Let $E_{i 1}, E_{i 2}, \ldots$ be the equivalence classes of $E_{i}$.

Let $m_{i}$ be the number of equivalence classes of $E_{i}$ and let $m_{0}=\max m_{i}$. Let $m=m_{0}$, except that $m=2$ if $m_{0}=1$ and there is an unbalanced loop at $v$. We define $\Psi$ to be the balanced graph with vertex set $(V \backslash\{v\}) \cup U$, where $U=$ $\left\{u_{1}, \ldots, u_{m}\right\}$ is disjoint from $V$, and edge set $E$ connected as follows: $\Psi$ restricted to $V \backslash\{v\}$ equals $\Phi \backslash v$; an edge $e \in E_{i j}$ is incident to $u_{j}$ instead of $v$, a balanced loop at $v$ becomes one at $u_{1}$, and an unbalanced loop at $v$ becomes a link from $u_{1}$ to $u_{2}$. Let $T$ be any tree spanning $U$ and let $\Phi^{\prime}=(\Psi \sqcup T) / T$.

We must show that $\Phi^{\prime}=\Phi$; then the proof will be complete. The underlying graphs are clearly the same and the loops at $v$ have the proper amount of balance. Consider a polygon $C$ at $v$, whose edges at $v$ are $e$ and $f$. Both $e$ and $f$ must lie in some $E_{i}$. If $e \sim f$, then $C$ is balanced in $\Phi$ so $e$ and $f$, in $\Psi$, are connected to the same vertex $u_{j}$. Then $C$ remains a balanced polygon in $\Psi$ and therefore remains balanced in $\Phi^{\prime}$. But if $e \nsim f$, then $e$ and $f$ are connected in $\Psi$ to different vertices, say $u_{j}$ and $u_{k}$ respectively. In $\Psi, C$ is an open path, completed to a polygon $D$ by the $u_{j} u_{k}$-path in $T$. Since $D$ is unbalanced, $C$ becomes, in $\Phi^{\prime}$, an unbalanced polygon. We are done, therefore, if we show that $C$ was unbalanced in $\Phi$; or, it suffices to say, in its subgraph $\Omega_{i} \cup(V,\{e, f\})$. In the latter graph, $\left\{w_{e}, w_{f}\right\}$ is a cutpair and $\Omega_{i}$ and the graph $\left(\left\{v, w_{e}, w_{f}\right\},\{e, f\}\right)$ are its bridges. Both bridges are balanced; thus by Lemma 1 , since $\Omega_{i} \cup(V,\{e, f\})$ is not balanced, $C$ is unbalanced in $\Phi$.

It is easy to see that $m$ is the smallest number of vertices that can be given to $T$ in the proposition.

A biased graph $\Phi$ is sign-biased if there is a sign labelling $\sigma: E \rightarrow\{+,-\}$ such that a polygon is in $B$ precisely when the product of its edge signs is + . By $[4$, 
Theorem 6], a necessary and sufficient condition for $\Phi$ to be sign-biased is that no theta graph have all its polygons unbalanced.

COROLLARY 1. If $\Phi$ is sign-biased and $\Phi \backslash v$ is balanced, then $|V(T)|$ can be taken to be 2 (or 1 if $\Phi$ is balanced).

Proof. Suppose in $\Omega_{1}$, say, there were three mutually inequivalent edges $e_{1}, e_{2}, e_{3}$. Let $P$ be a path in $\Omega_{1}$ from $e_{1}$ to $e_{2}$ and let $Q$ be a path in $\Omega_{1}$ from $e_{3}$ to $P$. Then $P \cup Q \cup\left\{e_{1}, e_{2}, e_{3}\right\}$ is a theta graph in which no polygon is balanced. This contradicts the cited result of [4].

We supplement this corollary with a converse.

Proposition 2. Suppose $\Phi$ is a biased graph of the form $(\Psi \sqcup T) / T$, where $\Psi$ and $T$ are as in Proposition 1. Then $\Phi$ is sign-biased if and only if $V(T)$ has no 3-bridge in $\Psi$.

PROOF. Let $v$ be the vertex in $\Phi$ corresponding to $V(T)$ in $\Psi$. Suppose there is no 3-bridge. Consider a theta graph in $\Phi$ composed of paths $P_{1}, P_{2}, P_{3}$ meeting at vertices $x$ and $y$. If $x, y \neq v$, the theta graph contains a balanced polygon composed of two paths not meeting $v$. If $y=v$, say, then in $\Psi$ at least two of the paths, say $P_{1}$ and $P_{2}$, end at the same vertex $t \in V(T)$. Then $P_{1} \cup P_{2}$ is balanced.

If there is a 3-bridge, it contains three internally disjoint paths from some vertex $x \notin V(T)$ to distinct vertices $t_{1}, t_{2}, t_{3} \in V(T)$. In $\Phi$ these paths form a theta graph having no balanced polygon.

One more remark about the case of Corollary 1 . Let $G(\Phi)$ be the bias matroid defined in [3] (for sign-biased $\Phi$ also see [5]). If $\Phi=(\Psi \sqcup T) / T$, where $\Psi$ is balanced and $T$ is an edge, then not only is $\Phi$ sign-biased, but even more $G(\Phi)$ is graphic, indeed isomorphic to the polygon matroid of $\Psi$. (For the proof see [6, Lemma 1F].)

The set of balancing vertices. Suppose $\Psi$ is a graph and $U \subseteq V(T)$. Let $W_{U}(\Psi)$ be the set of cutpoints $w \notin U$ such that $w$ separates all the members of $U$ in $\Psi$. (We are assuming $|U| \geq 2$.) We can now state our theorem.

THEOREM. Let $\Phi$ be a biased graph and $V^{*}$ its set of balancing vertices. Then $V^{*}=\varnothing$ except in the following cases:

(i) When $\Phi$ is balanced. Then $V^{*}=V$.

(ii) When $\Phi$ is unbalanced and has the form $(\Psi \sqcup T) / T$ where $\Psi$ and $T$ are balanced, $T$ is connected, and $V(T) \subseteq V(\Psi)$. Let $v$ be the vertex in $\Phi$ corresponding to $V(T)$. Supposing $V(T)$ has at least two 2-bridges in $\Psi$, then $\Phi$ has more than one unbalanced block and $V^{*}=\{v\}$. Supposing $V(T)$ has exactly one 2-bridge in $\Psi$, then $\Phi$ has just one unbalanced block and $V^{*}=\{v\} \cup W_{V(T)}(\Psi)$.

PROOF. Part (i) is obvious.

In part (ii) it is clear that each unbalanced block of $\Phi=(\Psi \sqcup T) / T$ arises from a 2-bridge, and vice versa. Note that $|V(T)| \geq 2$ because $\Phi$ is not balanced.

If $V(T)$ has at least two 2-bridges, then the only point common to every unbalanced block in $\Phi$ is $v$. Clearly, $\Phi \backslash v$ is balanced. Therefore, $V^{*}=\{v\}$.

If $V(T)$ has only one 2-bridge, again $v \in V^{*}$ but there may be other elements. For $w$ to belong to $V^{*}$ it is necessary and sufficient that in $\Psi \backslash w$ there is no path connecting two vertices of $T$. Thus, we have proved $\Phi$ behaves as described in part (ii). 
Now suppose $\Phi$ is an arbitrary unbalanced biased graph with $V_{1}(\Phi) \neq \varnothing$. Let $v \in V^{*}$. Then $\Phi=(\Psi \sqcup T) / T$ as described in part (ii), by Proposition 1. $V(T)$ must have at least one 2-bridge in $\Psi$, or else $\Phi$ would have been balanced. Thus we have $V^{*} \neq \varnothing$ precisely when the theorem says.

COROLlaRY 2. For a biased graph $\Phi$ we have $V^{*}=\varnothing$ except in the following cases:

(i) If $\Phi$ is balanced, $V^{*}=V$.

(ii) If $\Phi$ has exactly one unbalanced block, which is formed by 2-summing anywhere from 0 to $m$ balanced graphs into a contrabalanced graph $\left(m K_{2}, \varnothing\right)$ where $m \geq 3$, then $V^{*}=V\left(m K_{2}\right)$.

(iii) If $\Phi$ has exactly one unbalanced block, which is formed by 2-summing any number from 0 to $l$ of balanced graphs $\Phi_{i}$ (summed on the edge $e_{i} \in \Phi_{i}$ ) into an unbalanced polygon graph $\left(C_{l}, \varnothing\right)$ where $l \geq 2$, and if in each $\Phi_{i} \backslash e_{i}$ there is no cutpoint separating the endpoints of $e_{i}$, then $V^{*}=V\left(C_{l}\right)$.

(iv) If $\Phi$ has the form $(\Psi \sqcup T) / T$ as in Proposition 1 and $\Phi$ is not as described in (i), (ii), or (iii), then $\left|V^{*}\right|=1$.

A biased graph $\Phi$ constructed as in (ii) can be thought of as an unbalanced parallel connection of $m$ balanced graphs, some of which may be single edges. A $\Phi$ constructed as in (iii) can be regarded as an unbalanced series connection of $l$ balanced graphs, some of which may be single edges.

PROOF. The difference from the Theorem is in parts (ii) and (iii). We have to determine just when in part (ii) of the Theorem there is a point in $V^{*}$ besides $v$. Thus, $V(T)$ must have exactly one 2-bridge in $\Psi$ and $W_{V(T)}(\Psi)$ should be nonempty. We may as well assume that $\Psi$ is connected. Notice that $V(T)$ is stable in $\Psi$.

If $V(T)=\left\{u_{1}, \ldots, u_{m}\right\}, m \geq 3$, there can be only one point $w$ in $W_{V(T)}(\Psi)$ if it is not empty. Let the bridges of $\left\{w, u_{1}, \ldots, u_{m}\right\}$ be $\Psi_{i j}$ for $i=1, \ldots, m$, where $u_{i} \in V\left(\Psi_{i j}\right)$, and $\Psi_{j}$, where no $u_{i}$ is a vertex of $\Psi_{j}$. Let $\Phi_{1}$ be the union of all $\Psi_{1 j}$ and $\Psi_{j}$ and for $i \geq 2$ let $\Phi_{i}$ be the union of all $\Psi_{i j}$, with an additional link $e_{i}$, whose endpoints are $w$ and $u_{i}$, added to each $\Phi_{i}$ for $i=1, \ldots, m$. It is clear that $\Phi$ is obtained by 2 -summing each $\Phi_{i}$ at $e_{i}$ to a different edge of an $\left(m K_{2}, \varnothing\right)$ whose vertices are $w$ and $v$.

Suppose that $V(T)=\left\{u_{1}, u_{2}\right\}$. Then $W_{V(T)}(\Psi)$ can be arbitrarily large; say it equals $\left\{w_{2}, w_{3}, \ldots, w_{l}\right\}$ where $l \geq 2$, and let $w_{1}=v$ (corresponding in $\Phi$ to $\left\{u_{1}, u_{2}\right\}$ in $\Psi$ ). Evidently $\Psi$ has the form of a path $P$ whose vertices are, in order, $u_{1}, w_{2}, w_{3}, \ldots, w_{l}, u_{2}$, modified by 2 -summing into each edge $e_{i}^{\prime}$ (where, say, $e_{1}^{\prime}=u_{1} w_{2}, e_{i}^{\prime}=w_{i} w_{i+1}$ for $2 \leq i<l$, and $e_{l}^{\prime}=w_{l} u_{2}$ ) a suitable balanced graph $\Phi_{i}$ along its edge $e_{i}$. In order for the 2-sum to introduce no additional cutpoints separating $u_{1}$ from $u_{2}$ in $\Psi$, we must require that $\Phi_{i} \backslash e_{i}$ have no vertex separating the endpoints of $e_{i}$. Now since $T$ is a single edge and $(P \sqcup T) / T=\left(C_{l}, \varnothing\right)$ (as a biased graph), we can conclude that $\Phi$ is constructed as in (iii).

\section{REFERENCES}

1. W. T. Tutte, Graph theory, Encyclopedia of Math. and Its Appl., vol. 21, Addison-Wesley, Reading, Mass., 1984.

2. Th. Zaslavsky, Biased graphs, I. Bias, balance, and gains, J. Combin. Theory Ser. B (submitted). 
3. __ Biased graphs. II. The three matroids, J. Combin. Theory Ser. B (submitted).

4. _ Characterizations of signed graphs, J. Graph Theory 5 (1981), 401-406.

5. __ Signed graphs, Discrete Appl. Math. 4 (1982), 47-74. Erratum, ibid. 5 (1983), 248.

6. ___ Biased graphs whose matroids are special binary matroids (submitted).

Department of Mathematics, University of EVANSVille, EVANSVILle, INDiana 47702

Department of Mathematics, State University of New York, University Center at Binghamton, Binghamton, New YORK 13901 (Current address) 